Document downloaded from:

http://hdl.handle.net/10251/56588

This paper must be cited as:

Hellge, C.; Wiegand, T.; Guinea Torre, E.; David Gomez-Barquero; Schierl, T. (2013). Efficient HDTV and 3DTV services over DVB-T2 using Multiple PLPs with Layered Media. IEEE Communications Magazine. 51(10):76-82. doi:10.1109/MCOM.2013.6619569.

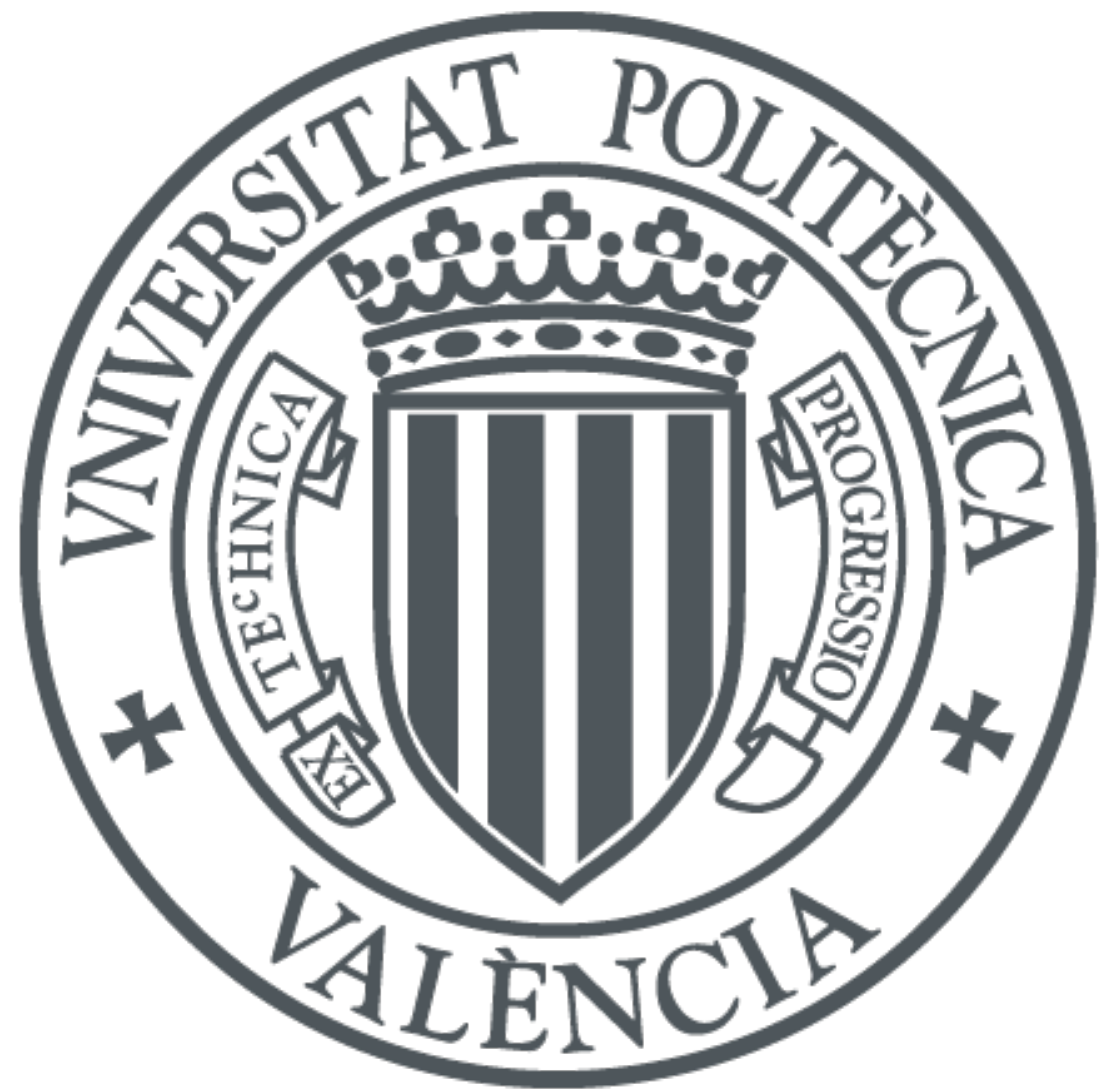

The final publication is available at

http://dx.doi.org/10.1109/MCOM.2013.6619569

Copyright Institute of Electrical and Electronics Engineers (IEEE)

Additional Information 


\title{
Efficient HDTV and 3DTV services over DVB-T2 using Multiple PLPs with Layered Media
}

\author{
Cornelius Hellge ${ }^{1,2}$, Estibaliz Guinea Torre ${ }^{1}$, David Gómez-Barquero ${ }^{1}$, Thomas Schierl ${ }^{1}$, \\ and Thomas Wiegand ${ }^{1,2}$ \\ ${ }^{1}$ Fraunhofer Institute for Telecommunications Heinrich-Hertz-Institute, \\ Image Processing Department, Multimedia Communication Group \\ Einsteinufer 37, 10587 Berlin, Germany \\ ${ }^{2}$ Technische Universität Berlin, Image Communications Group, \\ Straße des 17. Juni 135, 10623 Berlin, Germany
}

\{cornelius.hellge|estibaliz.guinea.torre|david.gomezbarquero|thomas.schierl|thomas.wiegand\}@hhi.fraunhofer.de

\begin{abstract}
The high bitrates of High-Definition or 3D-services require a huge share of the valuable terrestrial spectrum, especially when targeting wide coverage areas. This paper describes how to provide future services with the state-of-the-art digital terrestrial TV technology DVB-T2 in a flexible and cost-efficient way. The combination of layered media such as the scalable and $3 D$ extension of the H.264/AVC or the emerging H.265/HEVC format with the physical layer pipes feature of DVB-T2 enables flexible broadcast of services with differentiated protection of the quality layers. This opens up new ways of service provisioning such as graceful degradation for mobile or fixed reception. This paper shows how existing DVB-T2 and MPEG-2 Transport Stream mechanisms need to be configured for offering such services over DVB-T2. A detailed description on the setup of such services and the involved components is given.
\end{abstract}

Index Terms-3DTV, DVB-T2, HDTV, PLP, SVC, T2-Lite, MVC, SHVC.

\section{INTRODUCTION}

$\mathrm{D}$ VB-T2 [1] is the second generation digital terrestrial television technology that offers higher spectral efficiency, robustness and flexibility than any former digital terrestrial television system. DVB-T2 targets fixed and portable receivers and DVB-T2 lite targets mobile reception [2]. One of the main characteristics of DVB-T2 is the utilization of PLPs, which enable per-service specific robustness within the same DVB-T2 channel. Multiple PLPs allow adjusting the modulation, FEC code rate, and time interleaving of each delivered service to meet the target reception conditions. The usage of the PLP concept for service specific robustness of fixed and mobile services with single layer video coding has been analyzed in [3].

The multiple PLP feature of DVB-T2 fits very nicely with the layered media extensions of the H.264/AVC video coding standard, known as SVC [4] and MVC [5], or the upcoming scalable and 3D extensions of the H.265/HEVC format [6]. The combination of layered media formats with multiple PLPs presents a great potential to achieve an efficient and flexible provisioning of HDTV and 3DTV services in DVB-T2 systems. By transmitting the base representation using a heavily protected PLP and the enhancement representation in PLPs with less robustness but moderate/high spectral efficiency, it is possible to provide high quality services over a reduced and typically densely populated area, and provide standard quality services over a wider area as illustrated in Fig. 1. The same approach can be implemented by sending multiple representations of the same source at the same time, which is referred to as simulcast. Note that with simulcast, the same content is sent several times at different quality levels. I.e. in contrast to SVC, simulcasting does not remove the redundancy between the quality levels 


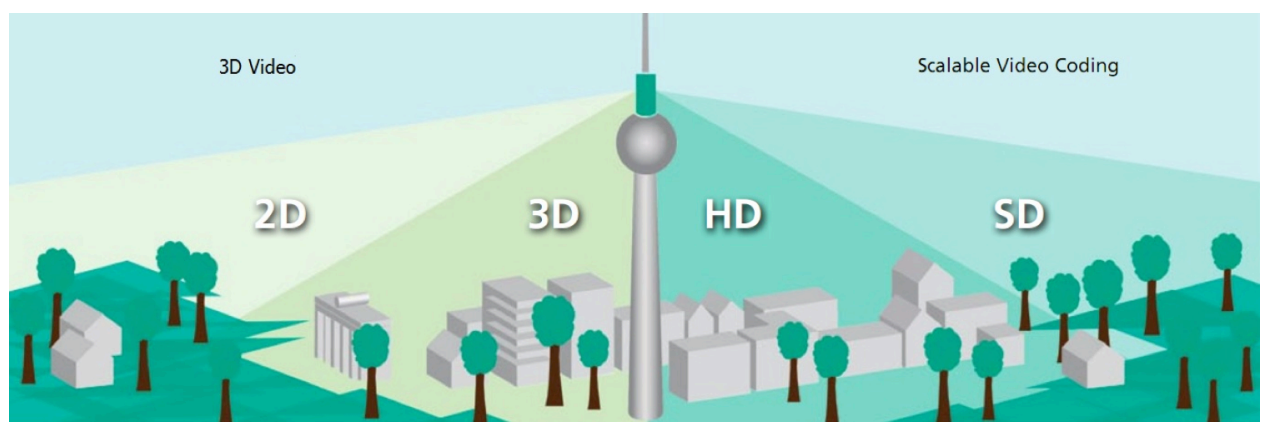

Fig. 1: Flexible provisioning of 3D services with MVC and 2D services with SVC

since both streams are encoded independently of each other. It must be also noted that the simulcast solution does not allow seamless switching between the qualities, as a receiver has to switch between the data PLPs. This leads to service interruptions of several seconds due to resynchronization to the new PLP, which is especially annoying in mobile reception where frequent switching can be expected. The potential of combining layered media formats with the multiple PLP concept of DVB-T2 has been shown in [7] by transmitting the media representations on different data PLPs. However, DVB-T2 receivers are only capable of decoding one single data PLP at a time [8], which makes such an approach not compatible to existing receivers.

On the other side, DVB-T2 receivers are able to process in parallel the common PLP, which is conceived to carry common signaling information shared by all data PLPs. This paper proposes to transmit layered media over DVB-T2 making use of the common PLP. Using the common PLP allows transmitting one representation within such PLP, while the other representation(s) can be allocated into a data PLP. The proposed approach fully relies on existing DVB-T2 mechanisms for MPEG-2 TS [9] splitting and recombining as specified in DVB-T2 for the separation and reconstruction of data to be carried in the common and the data PLP. In addition, the MPEG-2 TS standard provides all required means for signaling dependencies between the representations and hiding the enhancement representation from receivers only capable to process the base representation.

The rest of the paper is organized as follows. Table 1 introduces the abbreviations used throughout this paper. Section II gives an overview on layered video coding. Section III discusses the transport of SVC and MVC over MPEG-2 TS, and Section IV highlights the multiple PLP feature of DVB-T2. Section V describes in detail the proposed approach for combining layered media with multiple PLPs of DVB-T2, and Section VI discusses different reception use cases and their influence on the receiver behavior. Furthermore, Section VII shows analytical results in a mobile and fixed deployment scenario by comparing single layer deployment with SVC and simulcast.

\section{LAYERED VIDEO CODING}

Layered video codecs such as SVC or MVC, or the upcoming scalable and 3D extensions of the H.265/HEVC format allow for extracting different video representations from a single bit-stream, where the different sub-streams are referred to as representations or layers. The base representation provides the lowest level of quality and is a H.264/AVC or H.265/HEVC compliant bit-stream to ensure backwards-compatibility with receivers, which are only capable of decoding single layer streams. Each additional enhancement representation improves the video quality. For simplicity, from now on the paper will focus on the transmission of SVC. However it should be noted that all use case evaluations can be performed in the same way with MVC and future layered extensions of HEVC. SVC allows up to three different scalability dimensions: temporal, spatial, and quality scalability. SVC utilizes different temporal and inter-representation prediction methods for gaining coding efficiency while introducing dependencies between the different representations. Due to these dependencies, parts of the bit-stream are more important than others. A differentiation in robustness is in general beneficial for the transmission of layered codecs where the more important base representation gets a stronger protection than the enhancement representations. 
Table 1: Acronyms

\begin{tabular}{|l|l|}
\hline A & Audio \\
\hline AVC & Advanced Video Coding \\
\hline BL & Base Layer \\
\hline CBR & Constant Bit-Rate \\
\hline DVB-T2 & Digital Video Broadcasting - 2nd generation Terrestrial \\
\hline EL & Enhancement Layer \\
\hline FEC & Forward Error Correction \\
\hline HEVC & High Efficiency Video Coding \\
\hline HQ & High Quality \\
\hline LQ & Low Quality \\
\hline MODCOD & MODulation and CODing \\
\hline MVC & Multiview Video Coding \\
\hline PES & Packetized Elementary Stream \\
\hline PID & Packet IDentifier \\
\hline PLP & Physical Layer Pipe \\
\hline PMT & Program Map Table \\
\hline SI & Signaling Information \\
\hline SL & Single Layer \\
\hline SVC & Scalable Video Coding \\
\hline TS & MPEG-2 Transport Stream \\
\hline TSPS & Transport Stream Partial Stream \\
\hline TSPSC & Transport Stream Partial Stream Common \\
\hline VBR & Variable Bit-Rate \\
\hline
\end{tabular}

\section{MPEG-2 TS FOR SVC}

The MPEG-2 TS provides a standardized format for the transmission and storage of multimedia data. TS inherently provides all required signaling for real-time transmission, and is thereby perfectly suited for unidirectional video transmission. TS is specified as transport format for DVB-T2 [1] and is the widely used format in real world implementations. The transport of SVC in TS is specified by the latest amendments [9].

The key to transporting SVC over TS is the distribution of the representations to different PESs, which are indicated by different PIDs within the PMT. Each PID is associated with a "StreamType", which indicates the contained video profile. If the video profile is unknown to the receiver, the related TS is ignored. This allows for backward compatibility to legacy H.264/AVC capable receivers, which would only process the base representation of SVC. For deriving the dependencies between the media representations, the "Hierarchy Descriptor" is used to indicate the linking between the different representations.

Broadcast systems like DVB-T2 require input formats with CBR to allow synchronization of transmitter and receiver. In order to achieve the CBR, so-called NULL packets are inserted at TS multiplex generation. DVB-T2 allows removing the NULL packets before transmission (NULL Packet Deletion) and reinserting them after reception to avoid wasting valuable bandwidth while preserving compliance with the TS specification. Furthermore, this feature allows splitting of a full TS multiplex into so called partial TS streams, while keeping the bitrate of each partial TS constant.

\section{MultiPle PLPS IN DVB-T2}

The key features of DVB-T2 for enabling an improved spectral efficiency are thoroughly discussed by Vangelista et al. [1]. One of the main novelties of DVB-T2 is the introduction of PLPs to provide service-specific robustness. The DVB-T2 specification allows the constellation and FEC code rate, referred to as MODCOD, as well as the time interleaving duration to be assigned to each single PLP. Both the allocated capacity and the robustness of each PLP can be freely adjusted, such that it is possible to accommodate multiple use cases in the same frequency channel.

There are three types of PLPs: common PLPs, Data PLPs of type 1 and Data PLPs of type 2 . The data PLPs are intended to carry the actual T2 services. The common PLP is intended to carry data that is shared between several services, such as signaling, program guides or shared service components. The 
difference between the two types of data PLPs is the possibility of performing a feature known as subslicing which increases the time diversity.

Several PLPs can form a group of PLPs, which share one common PLP. Though the common PLP was originally conceived to transport common signaling data it can be used to deliver any type of data, as long as the receiver buffer model described in Annex C of the DVB-T2 specification is fulfilled [10]. Note that common and data PLPs share the same buffer and the constraints for the receiver buffer model also hold for the presented approach considering shared usage of the buffer by common and data PLP. This property can be used to efficiently transmit layered video services with two PLPs with differentiated protection, by defining one PLP group with one single data PLP.

The DVB-T2 specification [8] states that DVB-T2 receivers are only expected to decode one single data PLP. However, they must be able to decode up to two PLPs simultaneously when receiving a single service: the data PLP and its associated common PLP.

\section{MUlTiPle PLP DELIVERY For LAYERED MEDIA IN DVB-T2}

The key proposal of this paper is to carry layered media in two different PLPs: a data PLP and the associated common PLP inside a PLP group, with each PLP group associated with only a single data PLP. This way, the audio (A), base layer (BL), and signaling (SI) data can be carried in one PLP and the enhancement layer in the other PLP. This proposal admits both possibilities for the allocation of the media layers into either common or data PLPs. Considering that the common PLP is mapped to the frame in the same way as a type 1 PLP, it may benefit from less time interleaving than the data PLP if sub-slicing is employed. Therefore, it is recommended to use the data PLP to carry the A, BL, and the SI data with a robust MODCOD, whereas the common PLP carries only the EL data with a less robust MODCOD but a higher spectral efficiency. However, it should be noted that the option of transmitting the A, BL and SI in the common PLP and the EL in the data PLP is also valid, and the transmission procedure and mechanisms explained here also apply to this option. In any of the two approaches, the implementation is based on existing mechanisms in DVB-T2, MPEG-2 TS, and SVC specification.

The DVB-T2 standard [8] defines in its Annex D a mechanism to separate the data that will be carried in the data PLP from the data carried in the common PLP. This procedure consists of a splitting of the

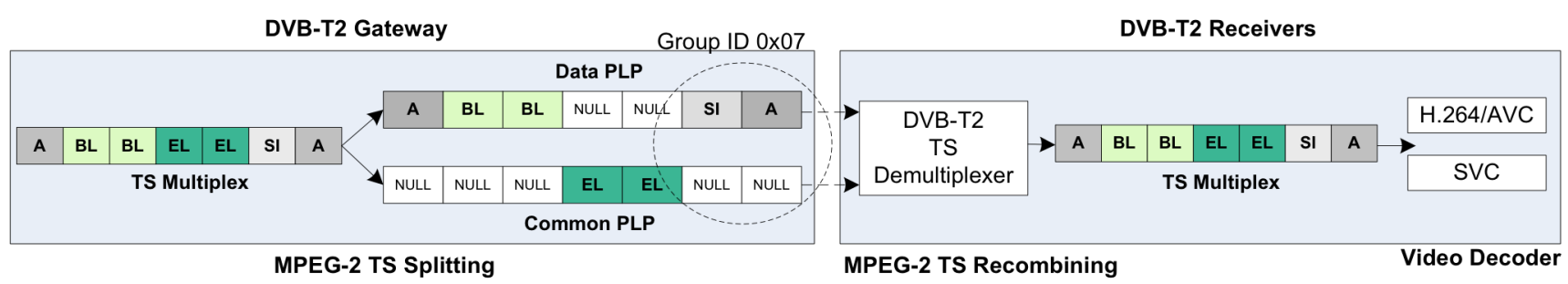

Fig. 2: DVB-T2 specified splitting and recombining of input MPEG-2 TSs into data PLP and common PLP of a group of PLPs

original TS multiplex, an allocation of packets to the dedicated PLPs, and a reconstruction of the original TS multiplex at the receiver. When a TS multiplex reaches the input of the DVB-T2 Gateway, the TS multiplex is split into one part going into a data PLP and the other part going into the common PLP. The partial streams are named TSPS and TSPSC. The information that is co-timed and identical in all of them is extracted and copied into the TSPSC at the same position. At the same time, the extracted packets are substituted by NULL packets in the corresponding TSPS. TSPSs and TSPSC are co-timed and have exactly the same bitrate, which is achieved by placing NULL packets in the TSPSC at position with no common data. The TSPSs are carried in data PLPs and the TSPSC is carried in a common PLP over the DVB-T2 system. At the DVB-T2 receiver, the original TS is reconstructed by doing a recombination of the partial streams, replacing the NULL packets in the TSPSs by the content of the TSPSC at the same time positions.

This mechanism can be easily extended to the representations of a layered media codec. Taking the above-explained procedure as a basis and adding a simple algorithm in the DVB-T2 gateway that extracts the representations by their corresponding PIDs, base representation packets can be allocated into the TSPSC and enhancement representation packets into TSPS, or vice versa. The procedure of the 
generation of common and data PLP and the recombining at the receiver is illustrated in Fig. 2. The most important implementation aspects of the proposal both in the transmission side (DVB-T2 Gateway) and reception side are explained in the following:

\section{A. Transmitter Implementation Aspects}

\section{MPEG-2 TS Stream Generation}

The media components of the service, A, BL, EL, are multiplexed together in a TS multiplex. For being transmitted over DVB-T2, this TS multiplex needs to have constant bit rate. This constant bit rate is achieved by the use of NULL packets. There can be multiple programs with multiple quality layers within a single TS multiplex. The TS signaling contains the PMT, which carries all the components, and their related PID.

\section{Stream Type Assignation}

According to the MPEG-2 TS specification [9], each stream is given a "stream type", which defines the type of MPEG-2 stream. The amendment 3 of this standard includes the type "SVC video sub-stream", which in this case is used for the enhancement layer. The base layer will be given the "AVC stream" type. This establishes the relation between the layers and makes the enhancement layer invisible to DVB-T2 legacy receivers not supporting SVC.

\section{Hierarchy Descriptor}

The linking between the base and the related enhancement layer is given by the "Hierarchy Descriptor" which is defined in the MPEG-2 TS [9]. The Hierarchy Descriptor gives information to identify the program elements that contain components of hierarchically coded audio or video. It allows identifying the base layer as such, and provides the hierarchical relation of the base layer with the corresponding enhancement layer.

\section{PLP Grouping}

DVB-T2 allows bundling a set of data PLPs into a group. Each group can have one associated common PLP that carries common information to all data PLPs. Each PLP group is identified by a group ID, which links the data PLP with its associated common. For the approach discussed within this paper, a PLP group with a single data PLP needs to be built. In this way, each PLP group can carry one or more layered video services.

\section{Splitting of the MPEG-2 TS Multiplex at DVB-T2 Gateway}

The TS multiplex is split in two partial TS like described in DVB-T2. After the generation of the partial TS streams, the DVB-T2 Gateway identifies the SI, A, BL, and EL packets. As previously explained in section III, for the data to be allocated in the corresponding PLPs the gateway replaces the A, BL, and SI packets in the data PLP (TSPS) and the EL packets in the common PLP (TSPSC) by NULL packets.

\section{B. Receiver Implementation Aspects}

\section{Selection of the PLP-Group}

When receiving the incoming information, the DVB-T2 receiver will first select the PLP group to be decoded. As previously mentioned, each group is identified by a group ID and contains one associated common PLP. The group ID is especially important for the receiver when more than one group of PLPs is present. In this case, it becomes necessary to link the data PLP to be decoded with the common PLP of the group where it belongs. By means of the group ID, the receiver will identify the data PLP that must be merged with the corresponding common PLP to recover the desired original TS multiplex.

\section{Service-decoding steps and TS recombining}

When the DVB-T2 receiver has selected the PLPs to be decoded, it first checks the PMT to find the PID of the service to be decoded. This table contains, for each program or service, the audio and video elements that belong to that service or program, as well as the PIDs of each program element. Afterwards, it will search the rest of the service components among the PLPs in the group, making use of the group ID again to identify and access the rest of the PLPs in the group. In the case of SVC, base 


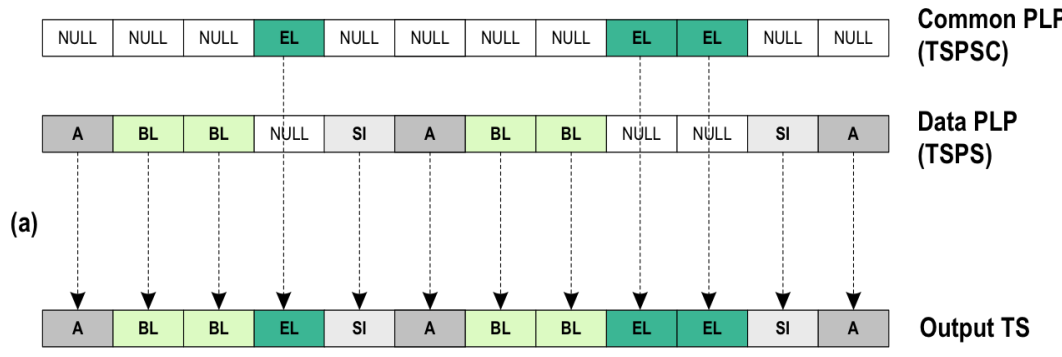

Fig. 3: Recombining of a SVC MPEG-2 TS with SVC enhancement layer delivered in the common PLP and base layer in the data PLP.

and enhancement layer packets will be given different PID values within the PMT. In the decoding process, the receiver identifies the dependencies among layers with the previously described "Hierarchy Descriptor". Finally, it merges the partial TSs and will forward the recovered TS multiplex to the video decoder.

\section{Transparency to H.264/AVC receivers}

SVC, MVC and their related transport format are designed in such a way that the base layer can be decoded by receivers that are only capable of decoding H.264/AVC. Such receivers are not aware of the "stream type" of the enhancement layer/view or the "Hierarchy Descriptor" and will ignore the related PIDs. They will only process the BL with the related audio and signaling. An SVC capable receiver is able to process both layers and is able to receive the higher quality.

As a summary, this procedure allows the transmission of SVC video services with differentiated robustness over DVB-T2 making use of the already existing MPEG-2 TS and DVB-T2 mechanisms. It does not require any change in existing devices and networks, and is transparent to current DVB-T2 receivers.

\section{ReCEPTION CONDITIONS: Use CASES}

For the use case described here, it is assumed that the data PLP carries the SVC base layer, signaling and audio and the common PLP carries the SVC enhancement layer. Once this is defined, different reception situations can happen: receivers can correctly decode both, data and common PLP, only the data PLP (the robustness of the data PLP should be higher than the common PLP), or none of them. In case both PLPs are correctly received, SVC receivers can reproduce the service with the highest quality. It should be noted that terminals equipped only with the H.264/AVC video codec couldn't make use of the information delivered via the common PLP. They can only reproduce the content delivered in the data PLP, which is H.264/AVC compliant. Naturally, in case only the data PLP is correctly received, receivers can only reproduce the H.264/AVC base layer of SVC.

TS Recombining when all PLPs are correctly received

In case both data PLP and common PLP are correctly received, DVB-T2 receivers should reconstruct the original TS by doing a recombination of the partial streams delivered by each PLP. It mainly consists of replacing the NULL packets in the TSPS by TS packets from the TSPSC at the same time positions. Fig. 3 illustrates this process.

\section{TS Recombining when only the Data PLP is correctly received}

In case only the data PLP is correctly received, DVB-T2 terminals cannot recover the full TS. However, it is possible to produce a syntactically correct TS based on the content delivered in the data PLP. The receiver can reproduce the TS delivered in the data PLP, but not the TS delivered in the common PLP. It is important to note that the reception of an erroneous PLP is currently not considered in the DVB-T2 implementation guidelines [11]. These guidelines do not describe the behavior of the DVB-T2 receiver when one of the PLPs contains errors. Currently no explanations are provided in the DVB-T2 specification on how the recombination should be performed when any of the PLPs turns out to be erroneous. Therefore, the way it is implemented depends on the manufacturer. 


\section{SVC FOR FIXED AND MobILE SERVICES}

One exemplary approach to analyze the performance of SVC is to calculate the cost in number of services that can be transmitted in a single DVB-T2 channel. This cost depends on the MODCOD settings of the scenario and the media bitrate allocated into the different PLPs. For this example two scenarios have been considered: one of them representing a typical fix-reception use case (A), and the other one belonging to a mobile reception scenario (B). The MODCOD details for each of the scenarios are shown in Table 2. In order to obtain a comparison, the number of transmitted services has been calculated for SVC, simulcast and SL cases. A SL video is used as reference case with a bitrate of 8 Mbps (e.g.720p@50Hz) for fixed reception and 2 Mbps (e.g. VGA@25Hz) for mobile reception, both sent with the highest robustness. For the simulcast case, the HQ stream has the same bitrate and quality as the SL. The low quality LQ stream is aligned with the SVC LQ. For the SVC case, coding penalties compared to single layer coding of $10 \%$ in the base and $10 \%$ in the enhancement layer have been assumed as similarly shown in [12]. Thereby, SVC and simulcast provide the same level of quality for HQ and LQ reception.

The required bandwidth per service for given MODCOD conditions and for a specific media bitrate can be calculated as follows. For each scenario, the spectral efficiency (bps/Hz) for the communication link is taken from Table 44 of the DVB-T2 implementation guideline [10] given for an FFT size of 8K, a guard interval of $1 / 32$, and a channel bandwidth of $8 \mathrm{MHz}$. As equation (1) shows, the bandwidth required by each SVC layer or simulcast stream $c$ in the channel is obtained from the division of the stream bit rate $R_{b}$ by the spectral efficiency $\eta$ of the PLP.

$$
c[H z]=R_{b}[b p s] / \eta\left[\frac{b p s}{H z}\right]
$$

The total bandwidth occupation of each service is obtained from the addition of the capacity requirements of the layers or streams. Finally, the number of services that can be transmitted results from the division of the channel bandwidth, $8 \mathrm{MHz}$, by the total bandwidth occupation.

Table 2 shows for exemplary service bitrates and for each use case the required bandwidth per service and the number of services that can be allocated within an $8 \mathrm{MHz}$ channel. Already the simulcast approach requires less bandwidth as SL, which can be further reduced by the use of SVC.

Table 2: MODCOD settings, video bitrates and bandwidth requirements for the different scenarios

\begin{tabular}{|c|c|c|c|c|}
\hline & \multicolumn{2}{|l|}{ Scenario A } & \multicolumn{2}{|l|}{ Scenario B } \\
\hline & Data PLP & Common PLP & Data PLP & Common PLP \\
\hline \multicolumn{5}{|l|}{ Multi PLPs } \\
\hline Modulation@Code rate(MODCOD) & 16 QAM@1/2 & 256 QAM@5/6 & QPSK@1/2 & 64@QAM 3/4 \\
\hline \multicolumn{5}{|l|}{ Simulcast } \\
\hline Video bitrates & $3.2 \mathrm{Mbps}$ & $8 \mathrm{Mbps}$ & $0.9 \mathrm{Mbps}$ & $2.0 \mathrm{Mbps}$ \\
\hline Required bandwidth per service & \multicolumn{2}{|c|}{$2.81 \mathrm{MHz}$} & \multicolumn{2}{|c|}{$1.36 \mathrm{MHz}$} \\
\hline Number of services in $8 \mathrm{MHz}$ & \multicolumn{2}{|c|}{2.84} & \multicolumn{2}{|c|}{5.90} \\
\hline \multicolumn{5}{|l|}{ SVC } \\
\hline Video bitrates & $3.5 \mathrm{Mbps}$ & $5.3 \mathrm{Mbps}$ & $1.0 \mathrm{Mbps}$ & $1.2 \mathrm{Mbps}$ \\
\hline Required bandwidth per service & \multicolumn{2}{|c|}{$2.56 \mathrm{MHz}$} & \multicolumn{2}{|c|}{$1.27 \mathrm{MHz}$} \\
\hline Number of services in $8 \mathrm{MHz}$ & \multicolumn{2}{|c|}{3.12} & \multicolumn{2}{|c|}{6.30} \\
\hline \multicolumn{5}{|l|}{ Single PLP } \\
\hline Modulation@Code rate(MODCOD) & 16 QAM 1/2 & - & QPSK 1/2 & - \\
\hline \multicolumn{5}{|l|}{ Single layer video } \\
\hline Video bitrates & $8.0 \mathrm{Mbps}$ & - & $2.0 \mathrm{Mbps}$ & \\
\hline Required bandwidth per service & $4.02 \mathrm{MHz}$ & - & $2.02 \mathrm{MHz}$ & \\
\hline Number of services in $8 \mathrm{MHz}$ & 1.99 & & 3.96 & \\
\hline
\end{tabular}

Fig.4 shows the number of additional services that can be transmitted, with respect to the single layer case, for SVC and simulcast and for a range of base layer ratios from $25 \%$ to $80 \%$. As can be seen from the results, the gain of SVC and simulcast heavily depends on the base layer ratio. The smaller the base layer or LQ stream, the higher the gain compared to SL. There is still a gain with $65 \%$ base layer ratio, 
which expected to provide a sufficient quality for the LQ case. Comparing SVC with simulcast, the gain of SVC increases with a higher base layer or LQ ratio, which is obvious due to simulcast needs to transmit the full bitrate for the LQ stream.

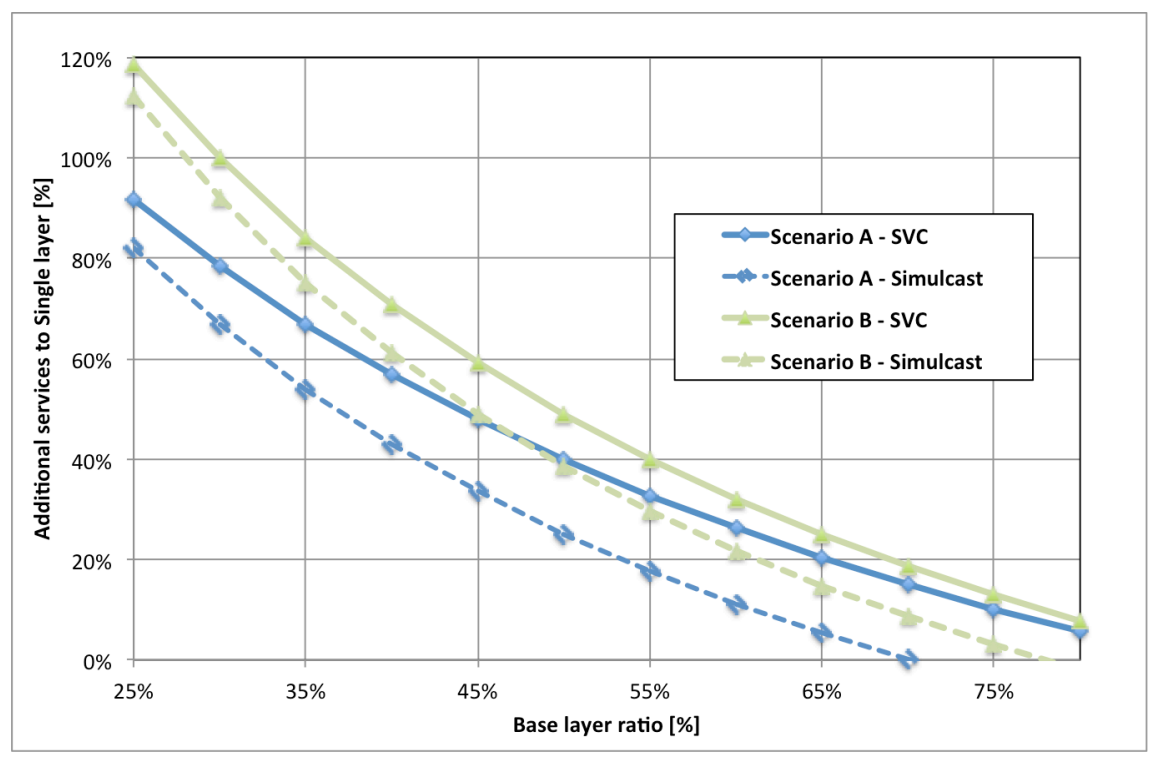

Fig. 4: $\mathrm{N}^{\circ}$ of additional services compared to single layer transmission for scenarios $\mathrm{A}$ and $\mathrm{B}$ for simulcast (without seamless switching) and SVC (with seamless switching)

\section{SUMMARY}

The combination of layered media codecs with multiple PLPs in DVB-T2 enables flexible and costefficient delivery of high quality HDTV and 3DTV services. This paper describes how to deliver layered media over DVB-T2 using multiple PLPs. The key is to use the common PLP to deliver one representation of the layered media stream, and transmit the second representations of the layered media stream in a data PLP. The proposed approach is fully compliant to the DVB-T2 specification. However, it needs to be noted that the behavior of legacy DVB-T2 receivers is not strictly defined in the case where one PLP cannot be decoded. Therefore, it depends on the receiver implementations how the more robust PLP is treated in such a situation. In general, the approach shows that multi-PLP delivery of data, which is already a feature of DVB-NGH, is possible also in DVB-T2 by means of the common PLP.

\section{REFERENCES}

[1] L. Vangelista et al., "Key technologies for next-generation terrestrial digital television standard DVB-T2, " IEEE Commun. Mag., vol. 47, no. 10, Oct. 2009, pp. 146-153.

[2] D. Gozalves et al., "DVB-T2 for Mobile and Mobile DVB-T2 (T2-Lite)," in Next Generation Mobile Broadcasting, Boca Raton, FL, USA: CRC Press, 2013, ch. 7, pp. 185-222.

[3] J. Morgade et al. "3DTV roll-out scenarios: A DVB-T2 approach," IEEE Trans. Broadcast., vol. 57, no. 2, June 2011, pp. 582-592.

[4] H. Schwarz et al., "Overview of the scalable video coding extension of the H.264/AVC standard," IEEE Trans. Circuits Syst. Video Technol., vol. 17, no. 9, Sept. 2007, pp. 1103-1120.

[5] A. Vetro et al., "Overview of the Stereo and Multiview Video Coding Extensions of the H.264/ MPEG-4 AVC Standard," Proc. IEEE, vol. 99, no. 4, Apr. 2011, pp. 626-642.

[6] G. J. Sullivan et al., "Overview of the High Efficiency Video Coding (HEVC) Standard," IEEE Trans. Circuits Syst. Video Technol., vol. 22, no. 12, Sept. 2012, pp. 1649-1668.

[7] L. Kondrad et al., "Cross-layer optimized transmission of H. 264/SVC streams over DVB-T2 broadcast system," Broadband Multimedia Systems and Broadcasting, 2009. BMSB'09. IEEE International Symposium on., Bilbao, Spain, May 2009, pp. 1-5.

[8] ETSI EN 302755 v1.2.1, "Digital Video Broadcasting (DVB); Frame structure channel coding and modulation for a second generation digital terrestrial television broadcasting system (DVB-T2)," Feb. 2011.

[9] T. Schierl et al., "Scalable video coding over RTP and MPEG-2 transport stream in broadcast and IPTV channels," Wireless Commun., vol. 16, no. 5, Oct. 2009, pp. 64-71.

[10] C. R. Nokes and O. P. Haffenden, "DVB-T2 Receiver Buffer Model (RBM): Theory \& Practice," BBC White Paper WHP 216, July 2012.

[11] ETSI TS 102831 v1.2.1, "Digital Video Broadcasting (DVB); Implementation guidelines for a second generation digital terrestrial television broadcasting system (DVB-T2)," Aug. 2012.

[12] H. Schwarz and T. Wiegand, "Further results for an rd-optimized multi-loop SVC encoder", JVT-W071, JVT Meeting San Jose, USA, 2007,ftp://avguest@ftp3.itu.int/jvt-site/2007_04_SanJose/JVT-W071.zip. 